\title{
User Modeling in Large Social Networks
}

\author{
Yuxiao Dong \\ Department of Computer Science and Engineering \\ Interdisciplinary Center for Network Science and Applications (iCeNSA) \\ University of Notre Dame, Notre Dame, IN 46556 \\ ydong1@nd.edu
}

\begin{abstract}
This proposal aims to harness the power of data, social, and network sciences to model user behavior in social networks. Specifically, we focus on individual users and investigate the interplay between their behavior and subsequently emergent social phenomena. Work in this proposal unveils the significant social strategies that are used by people to satisfy their social needs. We apply computational methods to address user modeling problems, including demographic inference, link recommendation, and social impact prediction. The proposed research work can be translated into applications in large social systems, such as mobile communication, online social media, and academic collaboration.
\end{abstract}

\section{Keywords}

user behavior; social impact; computational social science

\section{INTRODUCTION}

The interactions between individuals form the structural backbone of human societies, which manifest as networks. In a network sense, individuals matter in the ways in which their connections activate the emergence of new phenomena at larger, societal levels. In this proposal, we focus on modeling the various ways that different users are embedded in and interact within a network.

As the emergence of online social networking sites in the first years of the 21 st century, social network data has been explosively explored and studied. Essentially, a cornucopia of scientific advances, such as link prediction [9], influence maximization [8], community detection [7], and diffusion processes [10], have been and are being achieved by researchers from diverse communities. Indeed, social network research has developed itself as an interdisciplinary field, which attracts attention and simultaneously benefits from a variety of disciplines, such as data mining, sociology, physics, business and so on.

In this proposal, we aim to harness the power of social, data, and network sciences to model user behavior in social networks from multiple dimensions. Work on sociodemogrpahic modeling [5, 3] discovers that during the active dating period, younger users are active in broadening social connections with males and females alike,

Permission to make digital or hard copies of part or all of this work for personal or classroom use is granted without fee provided that copies are not made or distributed for profit or commercial advantage and that copies bear this notice and the full citation on the first page. Copyrights for third-party components of this work must be honored. For all other uses, contact the owner/author(s).

WSDM 2016 February 22-25, 2016, San Francisco, CA, USA

(C) 2016 Copyright held by the owner/author(s)

ACM ISBN 978-1-4503-3716-8/16/02

DOI: http://dx.doi.org/10.1145/2835776.2855087 while after reaching 35 years of age people tend to keep small, closed, and same-gender social circles. We also propose a data science model WhoAmI that demonstrates a greater than $80 \%$ potential predictability for inferring users' gender from phone call behavior and $73 \%$ for users' age from text messaging interactions. Work on modeling link formation $[6,4]$ studies the problem of link prediction in coupled networks, where we have the structure information of one source network and the interactions between this network and another target network, and aim to predict the missing links in the target network. We present a computational framework CoupledLP that is able to help a mobile operator achieve an accuracy of $80 \%$ for uncovering the top links of its competitor's network. Work on modeling social impact $[1,2]$ finds that in academic social networks, a researcher's authority on the publication topic and the venue in which a paper is published are crucial factors to the increase of his or her $h$-index, while the topic popularity and the co-authors' $h$-indices are of surprisingly little relevance. By leveraging relevant factors, we can predict whether a newly (previously) published paper will contribute to an author's future $h$-index with an $F_{1}$ score of 0.77 (0.99). To this end, modeling users in social networks offers the potential to understand the principles that underpin our highly connected world-from individuals, to communities, to societies.

Acknowledgments. The Ph.D. study is supported in part by the Army Research Laboratory under Cooperative Agreement Number W911NF-09-2-0053, the U.S. Air Force Office of Scientific Research (AFOSR) and the Defense Advanced Research Projects Agency (DARPA) grant \#FA9550-12-1-0405, and the National Science Foundation (NSF) Grants BCS-1229450 and IIS-1447795.

\section{REFERENCES}

[1] Y. Dong, R. A. Johnson, and N. V. Chawla. Will this paper increase your $h$-index? scientific impact prediction. In WSDM '15, pages 149-158, 2015.

[2] Y. Dong, R. A. Johnson, Y. Yang, and N. V. Chawla. Collaboration signatures reveal scientific impact. In ASONAM '15, pages 480-487, 2015.

[3] Y. Dong, J. Tang, T. Lou, B. Wu, and N. V. Chawla. How long will she call me? distribution, social theory and duration prediction. In Machine Learning and Knowledge Discovery in Databases, pages 16-31. Springer, 2013.

[4] Y. Dong, J. Tang, S. Wu, J. Tian, N. V. Chawla, J. Rao, and H. Cao. Link prediction and recommendation across heterogeneous social networks. In ICDM'12, pages 181-190, 2012.

[5] Y. Dong, Y. Yang, J. Tang, Y. Yang, and N. V. Chawla. Inferring user demographics and social strategies in mobile social networks. In $K D D^{\prime} 14$, pages 15-24, 2014

[6] Y. Dong, J. Zhang, J. Tang, N. V. Chawla, and B. Wang. Coupledlp: Link prediction in coupled networks. In $K D D^{\prime} 15$, pages 199-208, 2015.

[7] M. Girvan and M. E. J. Newman. Community structure in social and biological networks. PNAS, 99(12):7821-7826, 2002.

[8] D. Kempe, J. Kleinberg, and E. Tardos. Maximizing the spread of influence through a social network. In KDD'03, pages 137-146, 2003.

[9] D. Liben-Nowell and J. Kleinberg. The link prediction problem for social networks. In CIKM '03, pages 556-559, 2003.

[10] R. Pastor-Satorras and A. Vespignani. Epidemic spreading in scale-free networks. Physical review letters, 86(14):3200, 2001 